\title{
LA EDUCACIÓN SUPERIOR EN LA REGIÓN ANDINA: BOLIVIA, PERÚ Y ECUADOR
}

\author{
CRISTA Weise y José Luís LagunA*
}

\begin{abstract}
* Especialista en Evaluación y Acreditación de la Educación Superior. Profesora en la Universidad Mayor de San Simón, Cochabamba, Bolivia. Autora de la monografía sobre la educación superior en Bolivia publicada por IESALC-UNESCO. Ha publicado diversos artículos y capítulos de libros sobre la educación superior en Bolivia. Miembro del Grupo de Trabajo de CLACSO sobre Universidad y Sociedad. Email: c.weise@umss.edu.bo
\end{abstract}

Resumen: Este capítulo estudia en forma comparativa los sistemas de ES de Perú, Bolivia y Ecuador, que se caracterizan por un alto por ciento de población indígena y por su diversidad cultural y lingüística. Sus sistemas se han desarrollado de manera diversa.

Palabras Claves: Educación Superior. Sistemas de ES de Perú, Bolivia y Ecuador. Características sociohistóricas. Reformas Universitarias.

Abstract: This chapter discusses, from a comparative perspective the higher education systems of Peru, Bolivia and Ecuador. These systems are characterized by a high percentage of native Indian population and by their cultural and linguistic diversity. Their systems have evolved in different ways.

Keywords: Higher education. Higher education systems in Peru, Bolivia and Ecuador.

\section{Especificaciones para el análisis de los sistemas de educación superior.}

En este texto, ofrecemos una visión socio-histórica y organizacional, Ordorika (2001: 81) de la problemática universitaria que pone atención a las conexiones existentes entre los procesos que ocurren en el seno de la sociedad, los que tienen lugar en las Instituciones de Educación Superior (IES) en tanto instituciones de mediación cultural; y la interacción entre estos dos niveles. En segundo lugar, se trata de identificar los vínculos entre los objetos de carácter estructural con los de naturaleza cultural, reconociendo que las organizaciones de educación superior (ES) son espacios políticos en los que se confrontan visiones y proyectos educativos alternativos.

Las universidades del Perú, Bolivia y Ecuador no se han estructurado, ni aún en sus inicios, a partir de comunidades científicas sólidas o de tradiciones académicas fuertemente establecidas (Weise, 2005).

Entendemos que los procesos de cambio de la ES tienen que ver con disputas en torno a aspectos ideológicos, con los procesos histórico- sociales de constitución de los Estados, con la disputa alrededor de demandas contrapuestas (Carnoy y Levin, 1985 cit. en Ordorika, 2001), con la reproducción de la ideología, el acceso al conocimiento y, por lo tanto, a un capital cultural y simbólico de alto valor (Bourdieu, 1985 cit en Weise 2005) que posibilita el acceso a la 
igualdad social. Los proyectos de reforma universitaria son resultado de estas tensiones, muchas veces difíciles de reflejar a través de los datos disponibles sobre los sistemas.

Sin embargo, intentaremos movernos en esta perspectiva a lo largo de este texto, que si bien es de carácter descriptivo y pretende mostrar una visión panorámica de los sistemas, implica concebirlos como resultado de un proceso de negociación y conflicto que recoge intereses y visiones diversas y que es resultado de una dinámica de interacción entre las instituciones que conforman el campo de la ES en su desarrollo histórico.

\section{Características socio históricas. La construcción dinámica de los sistemas de ES.}

Podemos identificar al menos cuatro hitos históricos que marcaron el desarrollo constitutivo de la ES en esta región que se construyen en la dinámica universidad-Estado: I) el Estado Laico de principios del siglo XX ; II) las revoluciones nacionalistas de mitad de esa centuria, vinculadas a las luchas sociales en alianza con los movimientos estudiantiles; III) la implantación de las reformas neoliberales de finales de siglo, y; IV) la revolución cultural (indigenista y de las minorías) y de las comunicaciones globales de finales de los $80, \operatorname{los} 90$ y principios del nuevo siglo XXI.

El fracaso político y económico del nacionalismo revolucionario y los modelos de desarrollo estatal en América Latina, junto a la derrota política de los movimientos sociales y de las tendencias socialistas en todo el orbe, agravadas por la gran crisis económica de los años ochenta, impactaron en el rol de las universidades, convirtiéndose en correas transmisoras del nuevo pensamiento neoliberal privatizador y empresarial. (Weise,2005)

El resultado fue el incremento de las instituciones educativas privadas con regímenes de controles laxos y con ofertas curriculares ligadas al mercado alejadas de las necesidades sociales. Las instituciones públicas, a su vez, se vieron sobrepasadas por el crecimiento de la matrícula, y no lograron reestructurar su organización, configurándose como entidades complejas, pesadas y aisladas de la sociedad y el Estado.

Como resultado de las políticas de los 90, hoy tenemos sistemas diversificados, con diferentes grados de privatización y de redistribución de la matricula hacia el sector privado y un conjunto de políticas y reformas de corte muy similar encarnadas en los sistemas de educación superior de los tres países (Bolivia, Perú y Ecuador). 
Hacemos una descripción breve y sucinta de las principales características de cada uno de ellos, y aspectos en común y otros que los distinguen.

\section{El sistema Boliviano ${ }^{1}$}

\subsection{Características generales}

El sistema de ES en Bolivia, (LRE,1994) se compone de tres niveles: a) Universitario b) Tecnológico c) Normal.

Corresponde al primero la formación profesional en el ámbito de licenciatura y posgrado; al segundo, la de técnicos medios y superiores, y al tercero, la de profesores de educación primaria y secundaria.

En los hechos estos límites son un tanto borrosos. No se dispone de una delimitación clara y sancionada legalmente de los títulos que corresponde otorgar exclusivamente a cada institución, por lo que la anterior división es relativamente formal y se vuelve aún más difusa en nuevo Proyecto de Ley Avelino Siñani y Elizardo Pérez².

Es difícil hablar de un sistema universitario, si por ello se entiende un espacio coordinado y con cierto eslabonamiento y reconocimiento que facilite la movilidad docente y estudiantil. Tenemos más bien un conglomerado integrado por instituciones divididas por cuestiones históricas, legales y operativas. Esta situación se hizo más compleja en los 90 , cuando emergieron nuevos tipos de universidades, que conforman un conjunto de instituciones con orígenes y misiones, sistemas de gobierno y financiamiento distintos y hasta contrapuestas unas con otras. Estas instituciones mantienen una enconada rivalidad que impide incluso la transferencia de estudiantes del sector privado al público o el reconocimiento de los títulos de sus graduados. Paralelamente, la expansión del sector público continuó hacia regiones periféricas, distintas por tanto del eje central del país, hasta llegar a un total de 11 universidades públicas.

En Bolivia, la Universidad Católica Boliviana (UCB), fue hasta 1982 la única institución universitaria privada. Para 1990 operaban en el país seis universidades privadas.

En sus inicios las universidades privadas surgieron desde sectores religiosos o de los gremios empresariales, luego se convirtieron en atractivas inversiones

1 Basado en Rodríguez y Weise (2006)

2 La nueva Ley de Educación Avelino Siñani y Elizardo Pérez, les otorga a las normales el grado de Institutos Superiores alargando esta formación al nivel de licenciatura, les posibilidad de ofertar posgrados, al igual que a las universidades militares y propone la creación de las universidades indígenas como parte del sistema público de educación pero con carácter autofinanciado. 
educativas en manos de particulares en busca de rentabilidad y nichos de mercado no atendidos por ningún otro sector. En 1996 las universidades privadas "nuevas" eran ya 27 y disponían de 9 "subsedes" departamentales. Actualmente existen un total de 36 universidades públicas y 12 privadas.

En ese corto período de tiempo, la mayoría de las universidades privadas inició un importante crecimiento, pasando su matrícula de 26.901 estudiantes en 1996 a 56.764 en el 2002, llegando a doblarla.

Sin embargo, las instituciones privadas continúan siendo en general muy modestas. En el año 2000, la matrícula promedio rondaba entre los 600 a 2000 alumnos por universidad, mientras que en el promedio de las universidades públicas es de 40.000 a 50.000 alumnos.

A lo largo de este tiempo ha ido también disminuyendo el nivel de concentración del alumnado en la Universidad Mayor de San Andrés, la de mayor crecimiento en el país, que pasó de concentrar el 41,6\% de la matrícula del sistema público en 1972, a solo él 28\% del total de la matrícula en el 2002.

\subsubsection{Estructura normativa general.}

\section{Organismos de Coordinación Universitaria}

Las universidades públicas están reguladas por el "Estatuto Orgánico de la Universidad Boliviana", mientras que las universidades privadas tienen en el "Reglamento General de Universidades Privadas" (RGUP), aprobado por Decreto Supremo en el 2001, las disposiciones que norman su apertura, funcionamiento y evaluación. Se tiene dos estructuras normativas diferenciadas para cada sector y no se ha logrado compatibilizarlas ni integrarlas.

En los años 90 se organizaron unidades estatales destinadas a la regulación y la promoción de la ES, con el objeto de regular la proliferación de las universidades privadas ya instaladas, prácticamente de hecho y sin normativas previas consistentes.

El Viceministerio de Educación Superior, Ciencia y tecnología (VESCyT) tiene poca capacidad de interacción con la Universidad pública debido al principio de autonomía del que goza esta última.

Respecto al sector privado, el VESCyT cumple una función reguladora, vela por el cumplimiento de las normas e intenta el desarrollo de ciertas políticas para el sector.

Debido a la debilidad institucional del Estado, estas actividades de supervisión, regulación, y diseño de políticas son bastante limitadas y se encuentran muy permeadas por las relaciones de poder de grupos de interés ubicados fuera de la esfera estatal. (al respecto ver Weise: 2005) 
La Universidad Pública, por su parte, coordina sus actividades por intermedio del Comité Ejecutivo de la Universidad Boliviana (CEUB). Es responsable de representar a las universidades públicas, así como de coordinar, programar y relacionar sus actividades académicas, institucionales, administrativas y financieras. El CEUB no dispone de poder propio y por el contrario se halla sometido a las determinaciones de los Congresos Universitarios, máximos organismos del gobierno universitario, y a las Conferencias Nacionales Universitarias, por lo que algunos críticos ven en él una instancia superflua que no tiene competencia ni capacidad normativa para gestionar instituciones que gozan cada una de autonomía.

Las universidades privadas se encuentran agrupadas en la Asociación Nacional de Universidades Privadas (ANUP), Asociación Civil sin fines de lucro fundada en 17 de septiembre de 1990 y de afiliación voluntaria. En los hechos ANUP se ha revelado como un organismo más bien corporativo que académico. Están afiliadas a ANUP 22 de las 36 universidades privadas actualmente existentes.

\subsubsection{Caracterización de las universidades.}

Por su carácter, estas se pueden clasificar como Públicas y Privadas. En atención a la modalidad de sus mecanismos de coordinación con el gobierno central, existen dos tipos de universidades: a) Las Autónomas b) Las no Autónomas. Las primeras son aquella que deciden con libertad sus políticas académicas y de administración de recursos. Las segundas, en tanto, se hallan bajo inspección gubernamental, por mandato constitucional ${ }^{3}$.

Las Universidades Autónomas, son 11 públicas, ("iguales en Jerarquía" según el "Estatuto Orgánico de la Universidad Boliviana". De estas 11, 2 son de "Régimen Especial". Se distinguen de las anteriores porque no reciben financiamiento estatal y porque pertenecen a corporaciones definidas, una al Ejército y la otra a la Iglesia Católica). Las primeras (9) reciben una subvención anual "necesaria y suficiente" y figuran en el Presupuesto General de la Nación. Las segundas - la del Ejército y la Católica- en cambio, no pueden, por disposición constitucional, recibir ningún financiamiento estatal.

Actualmente podemos hablar de un nuevo tipo de universidad como son las universidades indígenas que en general están bajo el régimen privado, pero que son de carácter comunitario. La nueva ley Avelino Siñani prevé su creación como un tipo de universidad sui-generis cuyo régimen no está claro y que la ubica en un terreno poco preciso entre lo público-privado y su régimen autonómico o no.

3 Recientemente se ha creado la "Universidad Pedagógica", que es pública pero se halla bajo directo control gubernamental. 
Las universidades públicas establecieron desde 1931, un autogobierno de tipo colegiado. Este ha mantenido en escena siempre a los mismos actores: docentes y estudiantes, sin incluir en ningún momento a graduados ni administrativos, ni otro sector social externo a la comunidad universitaria ${ }^{4}$.

La situación cambió en el trienio 1953-1955, cuando las exigencias de democratización realizadas por el cuerpo estudiantil culminaron con la adopción de un sistema de Co -Gobierno, en el cual cada estamento tiene una representación y un poder equivalente al $50 \%$.

En los últimos años han arreciado las críticas contra este modelo de gobierno paritario docente estudiantil, tanto desde dentro como desde fuera de la universidad. Se lo califica de lento, ineficiente e incapaz de tomar decisiones trascendentes por el mutuo bloqueo gremial que genera una situación de empate y bloqueo.

Esta modalidad de gobierno no corresponde, sin embargo, a instituciones privadas autónomas o de Régimen Especial, donde la participación estudiantil es mucho más débil y las decisiones más piramidales y burocráticas. En estos casos los rectores no son electos sino designados por las instituciones corporativas.

Las Universidades Privadas en general han optado por un régimen de gobierno que privilegia a las autoridades unipersonales, principalmente el Rector. Estos son nombrados por los accionistas de la Universidad o en algunos casos por su Junta Directiva ${ }^{5}$.

\subsubsection{Prácticas de acceso, permanencia y cobertura de la ES.}

El sistema de ES boliviano es poco selectivo, el único requisito de ingreso es haber vencido el cuarto curso de secundaria en cualquiera de las modalidades del sistema educativo, con lo que se otorga el título de bachiller en humanidades; grado mínimo de escolarización que habilita para acceder a la ES universitaria. Existen sin embargo, diversas modalidades de acceso a la ES, de características diferenciadas entre el sector público y privado.

Hasta inicios de los 90, prevaleció en las universidades públicas el ingreso libre. A partir de allí, se comenzó a establecer de modo paulatino mecanismos selectivos, procedimiento normado a través de regulaciones generales emitidas por el CEUB, el estatuto orgánico de la Universidad Boliviana y de reglamen-

4 En una situación excepcional, en 1970 y 1971, algunas universidades, como la Tomás Frías de Potosí incorporaron a representantes de sindicatos fabriles, mineros y campesino, en una proporción paritaria a los sectores académicos. En esos mismos años en algunas universidades también existió un Co- Rector estudiantil.

5 En el Caso de la Universidad Católica Boliviana, el Rector es nombrado por la Conferencia Episcopal y el de la Escuela Militar de Ingeniería por el Comando General del Ejercito. 
taciones de carácter institucional y facultativo. En el caso de las universidades privadas, está normado por el RGUP, los estatutos orgánicos de cada institución y sus reglamentaciones internas.

En el sector público, el marco general de modalidades de ingreso reconoce cuatro mecanismos: Prueba de suficiencia académica, curso propedéutico, ingreso por excelencia e ingresos especiales.

En las Universidades Privadas predomina el ingreso libre. Si bien el RGUP señala la necesidad de establecer mecanismos de selección de la población estudiantil, la gran mayoría de instituciones aplica pruebas diagnósticas de carácter no selectivo, homogéneas para todas las carreras y facultades. Debido a la necesidad de competir por alumnos en el mercado, no se implementan pruebas selectivas, ya que éstas se sustentan casi exclusivamente con el cobro de matrículas estudiantiles.

No hay una prueba nacional de ingreso o Examen de Estado, y el Ministerio de Educación no interviene en este sentido. A partir de 1999 y de manera experimental se tomó una prueba nacional a bachilleres (Prueba de Aptitud Académica), cuyos resultados no son condicionantes para el ingreso a la ES y son las universidades las que determinan el uso de los resultados de las pruebas en el marco de sus propios procedimientos de acceso.

En Bolivia, las posibilidades de profesionalización se restringen casi exclusivamente a la ES universitaria. Las IES no universitarias tienen poco reconocimiento laboral, por lo que presentan menor demanda por parte de los jóvenes, son en su mayoría privadas y tanto la oferta como la cobertura son escasas. En contrapartida, el acceso a la educación universitaria es la principal aspiración de los jóvenes y familias de los diferentes estratos sociales, por lo que la demanda de ingreso en ella es muy alta (estimado entre un 75 y un $80 \%$ del total de bachilleres).

Sin duda esta situación, junto al crecimiento demográfico y el incremento de la cobertura escolar, explique por qué el porcentaje de estudiantes ha ido aumentando progresivamente, pese a la implementación de modalidades de ingreso. En 1990, la cobertura en relación al grupo de edad (17-24) era del 12,68\%. En 1992 ya alcanzaba el 13.72\% y en el 2000 llegó al 20,51\%. En el 2007 era de un $41 \%$. Tasa de cobertura superior a la media latinoamericana, pese a que en Bolivia, en los niveles primario y secundario, la cobertura es muy inferior a la de los otros países de la región. El sistema público en el 2002 acogía a 197.120 estudiantes universitarios $(76 \%)$ y a $74.286(23,75 \%)$ el privado, haciendo un total de 312.769 . El sistema boliviano se considera de tamaño medio y con un nivel bajo de privatización. (García Guadilla, 2002) 
No se cuenta con datos precisos sobre el tiempo promedio que toma un estudiante para graduarse, pero se calcula que más del $50 \%$ de los estudiantes que ingresan terminarían desertando. (World Bank; 1999, CEUB;1998 cit en Rodríguez y Weise,2007; CINDA, 2007)

Se calcula asimismo, que el tiempo promedio de estudio - entre 4 y 10 años en los 80 s y los 90 s - ha disminuido en los últimos años, como consecuencia de la flexibilización de las modalidades de graduación y en algunos casos de los planes de estudio.

Distribución de la matrícula por quintil de ingreso del hogar y tipo de institución.

\begin{tabular}{|r|r|r|r|r|r|r|}
\cline { 2 - 7 } \multicolumn{1}{c|}{} & \multicolumn{4}{|c|}{ Matriculados Carreras Universitarias } & \multicolumn{2}{c|}{$\begin{array}{c}\text { Matriculados Carreras } \\
\text { Técnico Superior }\end{array}$} \\
\hline $\begin{array}{c}\text { Quintiles } \\
\text { deingreso }\end{array}$ & $\begin{array}{c}\text { Privadas } \\
(2002)\end{array}$ & $\begin{array}{c}\text { Públs. } \\
(2002)\end{array}$ & $\begin{array}{c}\text { Total } \\
(2002)\end{array}$ & $\begin{array}{c}\text { Públs. } \\
(2000)\end{array}$ & $\begin{array}{c}\text { Total } \\
\text { Univs. } \\
(2001)\end{array}$ & $\begin{array}{c}\text { Total } \\
\text { Instits. } \\
(2001)\end{array}$ \\
\hline 1er (bajo) & 3,7 & 6,5 & 5,8 & 14,3 & 1,7 & 2,8 \\
\hline 2do. & 2,8 & 13,4 & 11,0 & 13,5 & 14,8 & 10,8 \\
\hline 3ro. & 10,6 & 19,8 & 17,7 & 17,4 & 11,4 & 18,0 \\
\hline 4to. & 13,7 & 25,4 & 22,7 & 23,2 & 50,4 & 26,0 \\
\hline 5to. (alto) & 69,2 & 34,9 & 42,8 & 31,6 & 21,7 & 42,5 \\
\hline Total & 100,0 & 100,0 & 100,0 & 100,0 & 100,0 & 100,0 \\
\hline
\end{tabular}

Fuente: Elaboración propia. Base: MECOVI, (2001-2002), INE (2002-2003)

Tomado de: CINDA, 2007

\subsection{Financiación}

Los datos nos muestran, a partir de los 90, un proceso de disminución del crecimiento de los fondos asignados a la Universidades públicas con algunos períodos dónde esto es más evidente. Asimismo, de 1990 al 2002, el Estado destinó en promedio un $1.25 \%$ del PIB a las Universidades públicas. (Ver: Lizárraga; 2002 y Santa Cruz, 2004)

En promedio, el $94 \%$ de los recursos totales asignados a las universidades se destinan al funcionamiento y tan sólo un escaso 6\% para la inversión. Esta situación, debido en buena parte a la elevada cifra de estudiantes que poseen las universidades públicas, limita la posibilidad de destinar fondos importantes a la actividad científica.

El costo per cápita promedio de la Universidad Boliviana disminuyó de 689,5 dólares por estudiante en 1997 a 498,7 en el 2000. 
Paralelamente, los ingresos por recursos propios han experimentado un incremento muy notable, elevando su participación en ingresos del 9\% (1986), al $25,07 \%$ (1995) y al 27,9\%(1998), aunque este crecimiento que proviene en general de la cooperación internacional (UMSS: 2003, CEUB: 2001), no ha tenido la misma intensidad en cada una de las Universidades públicas autónomas (Santa Cruz: 2004).

Los recursos públicos provienen de recaudaciones impositivas. Desde el 2005, las once universidades públicas coparticipan en el Impuesto Directo a Hidrocarburos (IDH). Se estima, que en un inicio, el monto alcanzará entre cuatro y cinco millones de dólares anuales, el que será distribuido entre todas las universidades del Sistema de acuerdo al criterio de población (no matrícula estudiantil). Estos recursos tienen destinos específicos: infraestructura, mejoramiento de la calidad, evaluación universitaria, investigación científica e interacción social y es probable que incida en las cifras que muestran el crecimiento del presupuesto general que se le asigna a las universidades.

Hasta 1990, se asignaba la subvención global proveniente del Presupuesto General de la Nación al sistema universitario. La Conferencia de Universidades establecía los coeficientes de reparto de estos fondos, mediante negociación entre las universidades. No se evaluaban los resultados del proceso educativo, sino que se negociaba en términos de los datos de matrícula.

A partir de ese año, se modificó el sistema de financiamiento, haciendo que las transferencias se negocien bilateralmente entre cada universidad y el Gobierno. Situación que permite que las universidades más grandes y situadas en regiones política y económicamente importantes dispongan de mayor poder de negociación, por lo que sus transferencias crecieron en la última década a tasas anuales dos o tres veces mayores que las tasas de crecimiento de las transferencias de las universidades pequeñas y de regiones periféricas (UDAPSO; 1993).

En un intento de cambio de perspectiva, en el 2001 se creó el "Fondo de Mejoramiento de la Calidad" (FOMCALIDAD), con el propósito de apoyar proyectos de reforma de los procesos de enseñanza-aprendizaje, de evaluación y de gestión institucional o de programas, y de abrir nuevas formas de financiamiento a las universidades, sin embargo esto no condujo al resultado esperado.

Las universidades públicas son gratuitas, mientras que el promedio del costo de la matrícula anual en las universidades privadas - en las que el costo fluctúa entre US\$ 300 y US\$2000 dólares anuales- es de US\$ 700. (VESCyT, 2002). 


\subsection{Reformas Universitarias, tendencias y experiencias}

Las reformas se han dado bajo la influencia de las políticas de los noventa con un acento fuerte en la innovación desde una perspectiva empresarial. Se acentuó la formación en gestión y los sistemas de gestión y evaluación y acreditación como elementos centrales. Se tomaron iniciativas como la línea de "innovación en gestión universitaria".

El intento de mayor envergadura, fue el Proyecto Inicial de Reforma de la ES, aprobado en el 2002, aunque varios de sus componentes estaban ya en marcha.

El proyecto comprendía dos programas centrales, el primero referido a la Modernización Institucional del Viceministerio y de las IES, y el segundo dirigido al mejoramiento de la Calidad de la ES, asentado principalmente en los mecanismos de evaluación y acreditación como sistemas de aseguramiento de la calidad (CONAES), cambio en las modalidades de financiación con el Fondo de mejoramiento de la Calidad (FOMCALIDAD), y restricción del acceso con las pruebas de aptitud académica. El propósito general del Proyecto inicial de Reforma era preparar las condiciones técnicas, políticas y sociales para el establecimiento del programa de Reforma de la ES y establecer mecanismos prioritarios que dinamicen procesos de mejoramiento sostenido de la calidad de la ES.

Con todo, muy poco se ha avanzado para transformar sus estructuras y el proyecto de reforma de la universidad boliviana aún no logrado tener una forma definida y clara. Muy pocas instituciones se han propuesto cambiar sus estructuras y han apostado al cambio y la innovación.

Quizá el caso más destacado por su seriedad con los compromisos asumidos resulta el de la Universidad Autónoma Juan Misael Saracho (UAJMS) que inició su proceso de reforma en 1998. Desde entonces se encara un proceso de modernización dentro el marco de la UNESCO cuyos postulados de pertinencia, calidad e internacionalización guían el proyecto de Cambio y Transformación, que ha sido nuevamente respaldado por UNESCO en el año 2007.

El modelo propone un conjunto integrado para el perfeccionamiento de distintos factores determinantes de la calidad, en este caso: conocimiento, organización, docentes, estudiantes, infraestructura y equipamiento, cooperación y relación internacional y gestión universitaria.

Otra iniciativa de reforma estructural relevante es la llevada a cabo en la UMSS, con apoyo de la cooperación Holandesa. 


\section{El sistema Peruano 6}

\subsection{Características generales}

La educación superior comprende los Institutos Superiores Tecnológicos, los Institutos Superiores Pedagógicos y las Universidades.

Los Institutos Superiores Tecnológicos y Pedagógicos, pertenecen a la jurisdicción del Ministerio de Educación, tanto en la autorización para su funcionamiento como en la supervisión y control del desarrollo educativo.

Las universidades del país, son públicas y privadas. Están regidas por la Ley Universitaria $\mathrm{N}^{\circ} 23733^{7}$ y el Decreto Legislativo $\mathrm{N}^{\circ} 882$, instrumentos normativos que marcan el escenario legal de interrelación Universidad-Estado. Las universidades públicas se crean por iniciativa del Estado, mientras que las universidades privadas por particulares.

En 1960 había una universidad privada, en 1969 eran 9, número que se mantuvo casi hasta los 80, mientras que entre 1980 y 2000 se crearon más de 33 universidades privadas. En el 2002 había 33 públicas y 45 privadas. En 2004 la cifra era de 35 públicas y 47 privadas para un total de 82 .

\subsubsection{Estructura Normativa General. Organismos de coordinación Universitaria.}

A diferencia de Bolivia, que nunca consensuó una Ley de ES, el Perú contó con una desde 1983, (LEY No 23733). El Decreto Legislativo $\mathrm{N}^{\circ} 882$ de 1996, norma la apertura y funcionamiento del sector privado, incluyendo aspectos relativos a posibilidades de organización, y particularmente a la posibilidad de constituirse en instituciones con o sin fines de lucro (no permitido en la legislación boliviana). De igual modo éstas pueden elegir bajo cual normativa regirse, ya sea bajo lo estipulado en la Ley o bajo la normativa que se establece en el Decreto Legislativo, lo que le da un amplio margen de acción.

El marco normativo legal peruano es común para todas las instituciones de ES, mientras que en Bolivia, existen dos normativas paralelas, ninguna con el rango de Ley.

Las universidades públicas peruanas son autónomas. Este derecho es extensible a las universidades privadas. La autonomía es de carácter académico, normativo y administrativo. (Ministerio de Educación, 2005)

6 Basado en los informes de país de IESALC en www.iesalc.org.ve

7 Emitida en diciembre de 1983. 
La autonomía tiene límites y alcances definidos. Como consecuencia de su régimen autonómico las universidades establecen sus normas y se autorregulan. Organizan su sistema académico, económico y administrativo; Administran sus bienes y rentas, elaboran su presupuesto y aplican sus fondos.

Para la regulación del sistema, se reconoce como órgano de coordinación a la Asamblea Nacional de Rectores (L. U. N N $^{\mathrm{O}} 23733$ ), compuesta por los rectores de las universidades públicas y privadas. Es un organismo nacional, estatal, y descentralizado de carácter autónomo.

Esta es considerada una de las máximas instancias de supervisión y regulación universitaria, pues incluso tiene la atribución de intervenir en las universidades públicas y privadas, cuando se presenten en ellas, graves irregularidades académicas, administrativas, normativas o económicas. Participa en asuntos relativos a la creación y supresión de universidades públicas y privadas, temas presupuestarios, de apertura y regulación de programas y titulaciones, de mediación entre las universidades y de publicación de estudios e informaciones sobre la ES peruana.

En el ámbito regional funcionan los Consejos Regionales Interuniversitarios, constituidos por los rectores de la región correspondiente. Los Consejos Regionales Interuniversitarios, funcionan de acuerdo a sus Reglamentos. Fundamentalmente emiten informes o dictámenes sobre aspectos específicos, solicitados por la Asamblea Nacional de Rectores.

\subsubsection{Caracterización de las universidades}

En el Perú, la ES está conformada por un conglomerado muy diverso de universidades. Del 2004 al 2008 la cifra de universidades pasó de 82 a 112, de las cuales 35 son públicas, 56 son privadas y 21 funcionan con una autorización provisional por 5 años. Entre las últimas encontramos universidades privadas no confesionales, confesionales o corporativas, en un contexto de ES altamente diversificado.

Dentro de las universidades privadas distinguimos aquellas que gozan de autonomía plena y las que están en proceso de consolidación y cuentan con autorización provisional y que tras el cumplimiento de ciertas regulaciones podrán adquirir su condición de autónomas plenas.

La forma de gobierno que se establece es el de gobierno por elección en una proporción de un tercio para autoridades, docentes y alumnos. Por el Artículo $\mathrm{N}^{\mathrm{o}} 42$ se permite a las universidades privadas representación diferente a los tercios. 


\subsubsection{Requisitos y prácticas de acceso, permanencia y cobertura demográfica.}

Al igual que en el caso de Bolivia, el Perú cuenta con un sistema de admisión regulado. Se establecen números clausus en cada facultad. No existen pruebas nacionales implementadas desde el Estado, si no que este procedimiento es definido en las propias universidades de acuerdo a su régimen interno y por lo tanto varía de una a otra. En las universidades privadas de igual manera está vigente un examen de ingreso que se rige por la normativa propia de cada una de ellas.

En el Perú la tasa bruta de matriculación es del 32,9\% (GUNI, 2008).

Resulta difícil saber cuántos de los jóvenes peruanos que ingresan a la universidad logran obtener el grado académico y en qué plazos. Vexler (2003) calcula que, considerando la matricula de ingreso en 1988 a 1997 y el número de graduados de 1993 a 2002, habrían concluido sus estudios en tiempo previsto el 55 \%, dando para 1989 un 34\%. De igual manera, Arregui (2002 cit. en Vexler, 2003) empleando como referente la relación de ingresantes y egresantes del mismo año, calcula en 5\% el porcentaje de graduados en 1989; este mismo porcentaje en el 2002 sería de $12 \%$. Ambos indicadores señalarían una mejora en los niveles de graduación, según Vexler (2003), en buena parte explicado por la participación de las universidades privadas.

\subsection{Financiación}

Son recursos económicos de las universidades públicas: las asignaciones provenientes del Tesoro Público, los ingresos por conceptos de leyes especiales y los ingresos propios.

Las universidades privadas tienen como fuente principal de ingresos los pagos de la matrícula de los estudiantes. Sin embargo, pueden recibir recursos estatales a través de algunos mecanismos especiales, a través de subsidios destinados en gran medida a becas.

La asignación presupuestaria sigue siendo de carácter incremental y negociado. Cada universidad pública elabora su presupuesto anual y lo remite a la Asamblea Nacional de Rectores (ANR).

Las asignaciones presupuestadas de cada universidad son determinadas por el poder legislativo, sobre la base de las propuestas y la información recibida. El Congreso de la República al aprobar el presupuesto anual del sector público, asigna al conjunto de las universidades un porcentaje del gasto corriente del mismo, considerando el incremento histórico. Los recursos son administrados 
en forma autónoma por las universidades, en coordinación con el ministerio de economía.

Las universidades privadas, tienen plena autonomía en el uso de sus recursos económicos.

Los estudios en las universidades públicas son gratuitos. Los estudiantes contribuyen con una suma casi simbólica por derecho de matrícula.

En el año 2003, se calculó el costo anual por alumno de la universidad pública en US\$1,373. (Gonzales de la Cuba, 2004)

\subsection{Reformas Universitarias, tendencias y experiencias}

Las reformas en el Perú tienen un corte similar a las realizadas en la región, inspiradas en las políticas reformistas de los 90, que como principales componentes incluyen: la creación de sistemas de acreditación y evaluación, los programas de mejoramiento de la gestión universitaria y las discusiones acerca de la regulación del ingreso y el arancelamiento de las universidades públicas, de manera casi idéntica a Bolivia y Ecuador.

Se señala que uno de los principales cambios ocurridos en la ES en Perú, es la elaboración, por parte de la Asamblea Nacional de Rectores, de un Plan de Modernización y Acreditación Integral, similar al proyecto de Reforma impulsado en Bolivia. Entre sus componentes vemos de manera coincidente propuestas de descentralización, acreditación universitaria, evaluación y autoevaluación. También proponen la implementación y desarrollo de las normas académicas ISO-9001, capacitación y actualización para la educación científica y tecnológica, la adaptación, actualización y modernización del currículo, coordinación, colaboración y cooperación intra e interinstitucional, y apoyo a la relación universidad - empresa.

Como podemos ver, en dichas propuestas se encuentra clara coincidencia con las políticas de modernización de los 90 que favorecen el desarrollo del sector privado y que ponen un fuerte énfasis en la regulación y la vinculación con la empresa.

En este proceso de reforma se planteó también: volver a involucrar al Estado y la sociedad civil con el destino y futuro de las universidades; elevar los requisitos para la constitución de nuevas universidades; y favorecer la participación de las mayorías nacionales en las universidades públicas y establecer elecciones universales y directas de autoridades.

Por otra parte, se propone la constitución de un órgano autónomo con las funciones de acreditación, supervisión y planificación universitaria, (CONUEA) similar al CONAES propuesto en Bolivia, que está en proceso de estudio. 
Otro elemento importante es el reforzamiento de la investigación y postgrado, asignándoles presupuestos diferenciados de las actividades regulares de funcionamiento.

Esta reforma también pretende impactar en el modo de financiamiento de la universidad pública, hoy automático y de sumas históricas, por otro, que combine lo histórico, con factores de pertinencia y productividad.

\section{La educación superior en el Ecuador}

\subsection{Características generales ${ }^{8}$}

El Sistema Nacional de ES, se integra por universidades, escuelas politécnicas y por institutos técnicos y tecnológicos, en los que se desarrolla la formación de nivel técnico superior, universitario y de postgrado.

Las Universidades y escuelas politécnicas son las responsables de la formación, en áreas profesionales y en las disciplinas científicas y tecnológicas.

Los Institutos superiores técnicos y tecnológicos, son establecimientos que orientan su función educativa a la formación en conocimientos técnicos o al fortalecimiento sistemático de habilidades y destrezas. Su ámbito es el de las carreras técnicas, tecnológicas, humanísticas, religiosas, pedagógicas y otras especialidades de post-bachillerato. Los institutos técnicos y tecnológicos otorgan títulos en el nivel técnico superior en las carreras correspondientes autorizadas por el Consejo Nacional de ES (CONESUP).

El Ecuador comparte características y problemas similares a los de los Bolivia y Perú: universidades masivas y populosas, recortes presupuestarios permanentes, pérdida de su rol social, filosófico y político, deterioro de la calidad académica, insuficiente investigación científica, baja remuneración docente, estructuras en crisis frente a las condiciones y desafíos de la sociedad del conocimiento global, presencia - cada vez más manifiesta- de población estudiantil de origen cultural diverso que no es valorada en su riqueza y aporte cultural.

En el Ecuador, uno de los rasgos más notables es la concentración de la matrícula en las universidades estatales de Quito y Guayaquil, situación que refleja el carácter polarizado del desarrollo económico y urbano. Otro de los fenómenos más resaltantes del Ecuador es el alto crecimiento de la matrícula, debido a la importante expansión económica del país, como resultado de la

8 Este texto está basado en los informe de Zurita, F, 2006, Pareja, 1986 e informes parciales de país www. iesalc.org.ve 
explotación petrolera iniciada en 1973 y que produjo en el eje central del País -Quito, Guayaquil y Cuenca -, un notable proceso de modernización y urbanización, que incrementó la demanda de servicios del estado, incluyendo la ES. En este período la ES tuvo un crecimiento de 18.7\% anual entre 1974 y 1978

y del $5 \%$ anual en la década de los 90's en la que las mujeres y las poblaciones indígenas llegan a tener una creciente participación. La creciente demanda fue afrontada parcialmente ampliando el sector privado.

En 1970 solo había un par de universidades privadas, En el período 19982002 el Congreso Nacional creó 12 universidades. Hoy existen 41 universidades privadas. De ellas, 9 son cofinanciadas por el estado y 32 son autofinanciadas. Las universidades públicas son 29 para un total de 70 .

\subsubsection{Estructura normativa general.}

Organismos de Coordinación universitaria.

De manera similar al caso peruano, la ES ecuatoriana rige sus acciones institucionales por la Constitución Política del Estado (Arts. 66, 74, 75, 76,79 y 161) y La Ley de ES que fue expedida por el Congreso Nacional el 13 de abril del año 2000 en un contexto polémico.

Las universidades - lo mismo públicas que privadas- están sujetas a la regulación estatal a partir de dicha Ley, cuya implementación ha tenido resistencias provenientes principalmente de la Universidad Nacional de Quito. Las universidades nacionales ecuatorianas y las privadas gozan de autonomía.

El órgano principal de supervisión es el CONESUP, conformado por nueve miembros de universidades y escuelas politécnicas públicas y privadas, del gobierno (Ministerio de Educación y CONACYT), de los Institutos Técnicos y Tecnológicos y de las Cámaras de la producción. En general desarrolla funciones de regulación y supervisión de las diferentes IES - públicas y privadas- en relación a aspectos administrativos, financieros y académicos.

5.1.2 Caracterización de las universidades.

A las Universidades y Escuelas Politécnicas se las clasifica en públicas y privadas; las primeras son aquellas que financia en su totalidad el Estado, en tanto que las particulares se clasifican a su vez en "cofinanciadas por el Estado" y "autofinanciadas". Las cofinanciadas, sin perder su calidad de privadas, reciben fondos del estado y por tales fondos deben responder ante los organismos de control del estado ecuatoriano. Las autofinanciadas no reciben ayuda directa alguna del Estado. Al igual que en Bolivia, no existe el lucro como fin dentro de las actividades de las Universidades. 
Todas las universidades del sistema ecuatoriano son autónomas y por lo tanto regulan su propio funcionamiento interno.

Como en Bolivia, existía en la Universidad Central de Quito el co-gobierno paritario docente-estudiantil, modalidad introducida a fines de los años 1960 pero que ha sido sustituida por la actual disposición que limita esa representación en la Asamblea y el Consejo Universitario, a un número equivalente al $50 \%$ de los docentes. Los trabajadores, por su parte, tienen una representación en ambos organismos equivalente al 10\% de la parte profesoral.

La elección de Rector y Vicerrector es universal, directa y secreta siendo la ponderación de los votos de profesores, estudiantes y trabajadores la misma que en los demás establecimientos universitarios y politécnicos.

En cuanto al régimen de organización académica no existen normas generales sobre esta materia y cada universidad es en principio libre de implantar el sistema que considere.

También en el caso del Ecuador se ha evidenciado una creciente demanda de las poblaciones indígenas por acceder a la $\mathrm{ES}^{9} \mathrm{y}$ se ha aprobado la primera universidad indígena intercultural (Amautay Wasy), que funciona bajo el régimen de universidad privada.

\subsubsection{Requisitos y prácticas de acceso,}

permanencia y cobertura demográfica

En la Ley de ES aprobada se establece como requisito de ingreso a la ES además del título de bachiller, haber cumplido con los requisitos del Sistema Nacional de Admisión y Nivelación, cuyo objetivo es seleccionar y mejorar el nivel educativo de los ingresantes a las universidades. Sin embargo, estas medidas no se han implementado hasta la fecha por lo que, en los hechos el ingreso a las universidades lo define cada institución a partir de su normativa específica.

Es así que hay universidades e institutos politécnicos que solicitan únicamente el título de bachiller, mientras que otras aplican diversos tipos de pruebas, vocacionales, psicotécnicas, de conocimientos, cursos de nivelación, propedéuticos, cursos preuniversitarios, exámenes de ingreso y otros.

En Ecuador no se establecen números clausus para el ingreso.

Entre 1974 y 1999, el número de ecuatorianos con instrucción universitaria se extendió del 3\% al 17\% en los mayores de 24 años.

Actualmente se calcula una tasa neta de cobertura de la ES en el Ecuador de un $20 \%$ del grupo de edad. Este acceso sin embargo es poco igualitario, como también sucede en Bolivia y Perú.

9 Al respecto Ver: el Informe sobre Educación y poblaciones Indígenas en el Ecuador, García F, 2007 en http://www.iesalc.org.ve 
Para el año 2000, la población universitaria alcanzaría a $198.887^{10}$, con lo que es considerado un sistema de tamaño mediano (García Guadilla,2002). La matrícula estaría así distribuida en un $70 \%$ para el sector público y un $30 \%$ para el sector privado.

\subsection{Financiamiento de la educación superior}

Las universidades públicas se sostienen en un 95\% con fondos públicos, mientras que entre las privadas tenemos universidades cofinanciadas por el Estado y universidades autofinanciadas. Las cofinanciadas reciben asignaciones parciales del Estado, y las autofinanciadas dependen de sus propios recursos. El procedimiento de asignación de recursos se rige por un sinnúmero de leyes, reglamentos y disposiciones especiales, que han llegado a constituir un sistema normativo de gran complejidad y variación entre instituciones. De hecho, ante la creciente crisis financiera de los establecimientos de ES, ellos constituyen un frecuente motivo de fricción y conflicto con el poder estatal. Los recursos de las universidades autofinanciadas provienen de aranceles, donaciones, servicios y otros.

El rubro más significativo son las subvenciones que el Estado otorga directamente a cada institución. Estas no se ajustan a ninguna norma general y dependen, en buena medida, de la capacidad de negociación y presión de cada universidad, frente al Ministerio de Finanzas y la Comisión de Presupuestos del Congreso Nacional. Se destinan fundamentalmente a gastos de operación el 78\% del total, apuntando este dato a otra dimensión de la crisis de las universidades ecuatorianas. En segundo término se encuentran aquellos fondos que provienen de rentas recaudadas regularmente por el Estado, en virtud de un conjunto heterogéneo de leyes y decretos especiales. Ejemplos de éstos son: un impuesto adicional a la renta del $11 \%$ que deben abonar todos las contribuyentes del país (del cual 10\% es asignado a los establecimientos oficiales y el $1 \%$ restante a los particulares), una participación en los intereses de la deuda pública interna, impuestos a las bebidas alcohólicas, etc. Un conjunto de normas impositivas particularmente significativo es el referido a la renta petrolera y agroexportadora.

Ecuador Invierte el alrededor del 1\% del PIB en ES, y entre el entre $20 \%$ y $30 \%$ del presupuesto total de educación. (García Guadilla, 2002)

10 Otras fuentes presentan datos divergentes, estableciendo una población cercana a los 300.000 estudiantes, así como también la distribución entre público y privado. (SIIES,2006) 
Las asignaciones a las universidades en el 2006 fueron de 34' 893.190 dólares; de este total corresponden $29^{\prime} 145.970$ a las universidades estatales y los restantes 3'235.527 a las universidades particulares cofinanciadas. Lo que resta del total, es presupuestado para funcionamiento del CONESUP y el CONEA, cifra que llega a un poco más de dos millones y medio de dólares.

\subsection{Reformas Universitarias, tendencias y experiencias.}

Las principales reformas vienen expresadas en la Ley de ES (2000), sin embargo, la aplicación de sus disposiciones recién ha comenzado. Las innovaciones tienen que ver -como también vimos en Perú y Bolivia- con rendición social de cuentas, ingreso, financiamiento y el establecimiento de contactos y relaciones directas con la comunidad. Sin embargo también incluye aspectos de organización interna tal como la reforma del sistema de gobierno.

Para los procesos de evaluación y acreditación, existe un sistema autónomo de evaluación y acreditación, que funciona en forma independiente, en cooperación y coordinación con el Consejo Nacional de ES (CONESUP).

El Sistema Nacional de Evaluación y Acreditación de la ES, según la Ley, lo dirige el Consejo Nacional de Evaluación y Acreditación de la ES (CONEA), el mismo que se establece como un organismo independiente, correlacionado con el CONESUP.

Las primeras evaluaciones ya fueron realizadas, siendo la primera en obtenerla la Universidad del Azuay, punta de lanza en el inicio de las reformas.

\section{Comparaciones sobre los sistemas ${ }^{11}$}

En términos de estructura los tres sistemas son similares, Bolivia tiene una estructura educativa con menos diferenciación, que desemboca directamente en la formación universitaria. Ecuador en cambio tiene una estructura más diferenciada que los otros dos países, con un desarrollo mayor de la formación profesional y tecnológica como salida formativa de nivel superior, poco contemplada en el sistema Boliviano.

La estructura de grados y el tiempo previsto de estudios es semejante, con la diferencia anteriormente anotada. La duración de las carreras de licenciatura e ingenierías se prevé entre 10 y 12 semestres, aunque en los tres casos los niveles de eficiencia interna no son los más óptimos y han constituido uno de los desafíos de las reformas.

11 Ver Anexo: cuadro 1 
Los tres países tienen estructuras estatales débiles y siguieron un curso histórico similar, en el que las universidades arrastraron la herencia colonial desde sus inicios y desarrollaron poco sus bases científicas, sufriendo de una crónica debilidad académica institucional.

Perú, golpeado por la violencia, con una autonomía debilitada por los largos períodos de intervención; Bolivia con un sistema de organización de universidades públicas más estructurado y por tanto con mayor capacidad de impactar en las políticas estatales; y Ecuador con un acceso tardío de su juventud a la educación superior y una alta concentración de la cobertura en Quito y Guayaquil.

Las políticas de reforma universitaria impulsadas en los 90 tuvieron el mismo corte y el mismo tipo de componentes, fueron implementadas o formalizadas de manera más tardía que en otros países de la región. Perú y Ecuador tienen un sistema unificado, bajo una misma normativa para el sector público y privado, mientras que Bolivia aún presenta fuertes resistencias hacia este tipo de figura, más aún a la de una Ley, que como vimos, en Perú y Ecuador son el referente normativo para la ES.

Como efecto de dichas políticas los sistemas fueron impactados, generando procesos de diversificación y expansión del sector privado; este fenómeno se dio de manera más aguda en el Perú que tiene poco menos de la mitad de su población universitaria en el sector privado y un conglomerado extremadamente diverso de 112 universidades. Bolivia, por su parte, ha mantenido una predominancia aún fuerte del sector público y aunque ha aumentado el número de universidades privadas, en relación a Ecuador, que tiene en total 70, y Perú, ha tenido un proceso de diversificación institucional más moderado.

Bolivia ha logrado mantener con carácter exclusivo el financiamiento estatal para el sector público, no ha modificado sus sistemas de gobierno y no ha llegado a implementar el CONAES, por lo que ha mantenido una distancia altamente resistente en relación a los intentos de regulación del Estado. En los otros dos casos (Perú y Ecuador), las reformas han tomado forma y cuerpo en organismos estatales y colegiados que regulan el sistema y que por otra parte contribuyen a la falta de diferenciación entre el sector público y privado, o más bien, al debilitamiento del sector público frente al sector privado, que sin embargo, no siempre tiene los niveles de calidad deseables.

Un aspecto común en Bolivia y Ecuador, vinculado al desarrollo y organización emergente de los movimientos populares e indígenas, es la aparición de las universidades indígenas y una clara demanda de atención de este sector educativo hacia sus necesidades, tema aún poco debatido y menos resuelto 
desde las políticas de ES de la región, que inspiran sus reformas en paradigmas muy alejados de estas preocupaciones.

Los sistemas universitarios de Bolivia y Ecuador son pequeños y el de Perú mediano. Bolivia y Perú se ubican cerca de la media latinoamericana de tasa bruta de matrícula y Ecuador está por debajo de ella. Es evidente que el Perú es el país de entre los tres, que tiene un sistema con mayor grado de exclusión y privatización y se sitúa en un puesto bajo de inversión en educación en relación a los países de la región.

Las universidades andinas, comparten problemas similares a las de todos los países latinoamericanos: masificación, debilidad estructural, funcionamiento interno caótico y politizado, y pérdida de legitimidad, entre otros aspectos; sin embargo, cada una de ellas mantiene ciertas características particulares en sus relaciones con el Estado y que han dado -como resultado de las capacidades de construcción y negociación de sus actores- cierto tipo de sistemas, unos con presencia estatal más fuerte, como en el Perú, otras con el sistema o la organización del sector público más fuerte como es el caso Boliviano y otros con un peso importante de penetración del sector privado y empresarial al mundo universitario como en Ecuador, cuyas universidades públicas han perdido mucha legitimidad.

Si bien en todos los países se reconoce la autonomía en sus constituciones, cada régimen nacional tiene particularidades. Por ejemplo, la Autonomía universitaria en Bolivia ofrece una abierta posibilidad para que las políticas académicas y financieras se ejecuten por iniciativa casi exclusiva del sistema universitario y en muchos casos de cada centro académico, bajo un régimen de rendición de cuentas laxo y estrictamente financiero. La autonomía está sobreentendida como un régimen de no intervención en el que el sistema universitario público diseña y opera sus políticas de forma independiente del Estado, su relación con él es principalmente financiera, sin influir directamente en sus políticas. En términos estructurales prácticamente se ha mantenido intocada desde la reforma de Córdoba. Este es un caso extremo, en el que la autonomía ha servido de paraguas para oscurecer los problemas académicos universitarios y aislar a la universidad de la sociedad, al contrario de sus finalidades manifiestas.

En Ecuador, el Estado ha desarrollado más su rol regulador y ha cedido importantes espacios al sector privado, al igual que en Perú. El CONUEP tiene directa responsabilidad de las políticas académicas supervisa y autoriza inclusive la ejecución de nuevos programas académicos, desde donde se define también su participación del régimen fiscal; por otra parte, el Ministerio de Hacienda negocia directamente con cada centro académico su presupuesto, eso 
se hace en términos de la capacidad política de las autoridades. Por lo que la autonomía está circunscrita al régimen interno de las universidades públicas y privadas (elección de autoridades, administración de sus recursos, diseño y propuesta de programas académicos, entre otros).

De igual modo, la ley en el Perú reconoce la autonomía y como en Ecuador, establece límites a través de las instancias de coordinación. Sin embargo, cabe señalar que la Universidad peruana ha pasado por largos períodos de intervención y de restricción de la autonomía, durante el periodo de violencia vivido con la presencia de la guerrilla maoísta de Sendero Luminoso y el terrorismo de Estado desarrollado para combatirla. (Laguna, 2007) Este conflicto afectó fuertemente la universidad peruana, y la reconstitución del régimen autonómico peruano hasta la fecha no encuentra vías para consolidarse y remediar los daños que esta situación ha ocasionado en toda su potencialidad académica e institucional que hasta la década del setenta había sido un paradigma en Sud América.

En general, las reformas desarrolladas no han logrado un verdadero mejoramiento de la calidad, y los componentes desarrollados están, en estos países, en estado bastante embrionario. No se han modificado las formas de financiamiento, los sistemas de regulación no están logrando del todo garantizar la calidad, y no se han implementado las pruebas de admisión nacional.

Sin embargo, en los tres países - en universidades puntuales- se evidencia una notable mejora en sus niveles de cobertura, de eficiencia interna y en los esfuerzos de innovación y reforma. Dentro de estos avances es importante mencionar los esfuerzos por la incorporación de nuevas tecnologías, y los procesos de transformación académica e institucional que se vienen desarrollando, como norma, por iniciativa de las propias universidades.

A diferencia de otros países de la región, como Argentina, Brasil o Chile, los países Andinos se han mantenido más apegados al espíritu del modelo de la Universidad de Salamanca, que cumplió un rol más político e ideológico que de producción científica o académica.

Pese a los avances, parece que el tenor de las reformas actuales resulta insuficiente, incluso extemporáneo para una sociedad que ya ha cuestionado fuertemente el fundamento de estas políticas. Ante su fracaso, se está cambiando de norte hacia la búsqueda de modelos más integradores y democráticos. En este escenario de emergencia de los movimientos sociales, de crecimiento de la demanda y del valor de la educación en la sociedad, de la necesidad de construir su sentido de bien público, de generar visiones renovadoras de reconocimiento intra e intercultural, no se ha logrado orientar adecuadamente las 
políticas universitarias hacia fines más claros y comprometidos de pertinencia y justicia social.

Tampoco es posible sostener que los actuales sistemas están desarrollando una actividad pertinente a las demandas actuales, ni en cuanto a sus ofertas formativas, ni en cuanto al tipo de formación brindada, que sigue centrada en la transmisión de conocimientos y no en el desarrollo de capacidades adecuadas para las necesidades de las complejas sociedades contemporáneas andinas, teñidas de colores y sabores remotos, de identidad mestiza y textura cultural abigarrada.

En medio de un evidente cambio de las políticas de los gobiernos, con procesos de reconstitución social y de transformación de las relaciones de poder fuertes, en el caso boliviano; y de medidas populistas de retorno al Estado protector que predominan en Ecuador y Perú, el papel de las IES queda diluido. Quedan asignaturas pendientes para hacer frente a los desafíos de la integración cultural, la integración de saberes, la inclusión social, el desarrollo económico y el verdadero mejoramiento de la calidad de vida de los habitantes de los Andes.

Desde las reformas presentes en los sistemas de ES, no se ha logrado (ni pretendido) remover las estructuras universitarias, cambiar su enfoque e impactar en el mejoramiento de la calidad de la formación de los profesionales con un sentido de pertinencia sociocultural.

En este marco, las IES deberán repensar no sólo sus estructuras internas, si no particularmente su rol social y ello pasa por restituir sus formas de relación e interacción con las comunidades locales y nacional, fortaleciendo su función académica, generando comunidades de conocimiento. Encarando el desafío de recuperar su carácter protagónico, posicionándose en su realidad. La de los países andinos, naciones que deben asumir su diversidad como un valor constitutivo y que se mueven en la marea de influencias de origen remoto y a la vez actual, exógeno y a la vez local, región en todo sentido mestiza, diversa y única. 
Anexo 1 Cuadro Comparativo. Características de los Sistemas de ES. Bolivia, Perú y Ecuador ${ }^{12}$

\begin{tabular}{|c|c|c|c|}
\hline & Bolivia & Perú & Ecuador \\
\hline $\begin{array}{l}\text { Número de universi- } \\
\text { dades (2002) }\end{array}$ & $\begin{array}{l}48 \\
\text { (36 públicas y } \\
12 \text { privadas) }\end{array}$ & $\begin{array}{l}112 \\
\text { (35 públicas, } 56 \text { priva- } \\
\text { das, y } 21 \text { en proceso } \\
\text { de ser reconocidas) }\end{array}$ & $\begin{array}{l}70 \\
\text { (29 públicas } y \\
41 \text { privadas) }\end{array}$ \\
\hline $\begin{array}{l}\text { Matrícula privada } \\
\text { (2002) }\end{array}$ & $19 \%$ & $40 \%$ & $30 \%$ \\
\hline $\begin{array}{l}\text { Tasa Bruta de } \\
\text { Matrícula. }\end{array}$ & $41 \%$ & $33 \%$ & $20 \%$ \\
\hline Tamaño del sistema & $\begin{array}{l}312,769 \text { estudian- } \\
\text { tes. Mediano }\end{array}$ & $\begin{array}{l}450.000 \text { estudiantes } \\
\text { Grande }\end{array}$ & $\begin{array}{l}198.887 \text { estudiantes } \\
\text { Mediano }\end{array}$ \\
\hline $\begin{array}{l}\% \text { del presupuesto } \\
\text { de la ES con relación } \\
\text { al PIB }\end{array}$ & 1,25 & $0,5 \%$ & $1 \%$ \\
\hline $\begin{array}{l}\text { Costo por alumno } \\
\text { en US\$ }\end{array}$ & 498,7 & 1.373 & \\
\hline $\begin{array}{l}\text { \% del presupuesto } \\
\text { total de educación }\end{array}$ & Menos del $20 \%$ & $30 \%$ & $20-30 \%$ \\
\hline Tipo de asignación & $\begin{array}{l}\text { Histórica incre- } \\
\text { mental }\end{array}$ & Histórica Incremental & Histórica incremental \\
\hline $\begin{array}{l}\text { Organismo de } \\
\text { evaluación }\end{array}$ & $\begin{array}{l}\text { CONAES (no } \\
\text { implementado) }\end{array}$ & $\begin{array}{l}\text { CONEAU En proceso } \\
\text { de definición }\end{array}$ & CONEA \\
\hline Autonomía & $\begin{array}{l}\text { En públicas úni- } \\
\text { camente. Con } \\
\text { mayor alcance y } \\
\text { sin restricciones. }\end{array}$ & $\begin{array}{l}\text { En públicas y priva- } \\
\text { das. Recientemente } \\
\text { restituida. Con alcan- } \\
\text { ce interno y con res- } \\
\text { tricciones que derivan } \\
\text { de la Ley. }\end{array}$ & $\begin{array}{l}\text { En públicas y privadas. } \\
\text { Con alcance interno. Su- } \\
\text { peditada a la Ley. }\end{array}$ \\
\hline Gobierno & $\begin{array}{l}\text { Paritario docente } \\
\text { estudiantil. }\end{array}$ & $\begin{array}{l}\text { Definido en la Ley. } \\
\text { Cogobierno tripartito. } \\
\text { Autoridades, docentes } \\
\text { y estudiantes. }\end{array}$ & $\begin{array}{l}\text { Tripartito en la Universi- } \\
\text { dad Nacional de Quito, } \\
\text { con participación de ad- } \\
\text { ministrativos. Definido en } \\
\text { cada universidad. }\end{array}$ \\
\hline
\end{tabular}

12 Según las cifras del Instituto de Estadísticas de UNESCO para el 2005, en el caso del Perú, el sistema había alcanzado la cifra de 909,315 alumnos, mientras que en Bolivia la cifra era de 346,056 alumnos. En el caso de Ecuador no aparecen cifras reportadas. (UNESCO, 2007, p. 128) 


\section{Bibliografía}

Carnoy M. y Levin, H. (1985). Schooling and Work in the democratic State. Stanford University Press. Stanford.

CINDA (2007). Educación Superior en Iberoamérica Informe 2007. Santiago, Chile

CONEA, (2004).Oferta académica de Educación Superior en el ecuador. Estudio comparativo de títulos. CONEA. Quito Ecuador

Cordero Iñiguez, J. (2003). Reflexiones sobre la educación superior ecuatoriana. La Educación Superior ecuatoriana en los últimos cinco años: 1998 - 2003. Quito.

Esquetini Cáceres C. Rodríguez, E. (2006). Estructura y titulaciones de Educación Superior en Ecuador http://www.oei.es/quipu/ecuador/index.html

González de la Cuba, J. (2004).R. El financiamiento de la educación superior en el Perú. Lima. Consultado en (22.01.08) http://www.iesalc.unesco.org.ve GUNI (2008) La Educación Superior en el Mundo 3. Mundi-Prensa, Madrid. Informe nacional de educación superior en el Perú (2003) en http://unesdoc. unesco.org/images/0013/001316/131677s.pdf

Informe Nacional sobre la Universidad Peruana (2005). Razones para una reforma universitaria Ministerio de Educación. Oficina de Coordinación Universitaria Nacional de la ES en Perú. Consultado en (22.01.08) http://www. iesalc.unesco.org.ve

Asociación Nacional de Rectores. (2002) Informe sobre educación superior Universitaria Lima Perú Consultado en (22.01.08) http://www.iesalc.unesco. org.ve

IPP (2005) balance de la educación peruana: Avances en el papel, marcando el paso en resultados. Consultado en (18.01.08) http://www.ipp-peru.com/upload/ INFORME-47-2006.pdf

Laguna, J.L. (2006). "Pueblos originarios, interculturalidad y educación superior". Ponencia presentada al Seminario "Universidad y Culturas. Reflexiones sobre la Descolonización de la educación Superior. Universidad Mayor de San Simón.

Mollis, M. (2001). La universidad argentina en tránsito. Fondo de Cultura económica. Mexico.

Moreno A. (s.f.). Universidad crisis y reforma" en Situación y Desarrollo de la Universidad. Universidad Central. Quito. 
Nava L. (2003) Evaluación y Acreditación de la educación superior: El Caso Del Perú en http://tarwi.lamolina.edu.pe/ nava/Caso\%20Peru.pdf

Ordorika, I. (2001) Aproximaciones teóricas para el análisis del conflicto y el poder en la educación superior. Vol. XXIII. N 91. Perfiles Educativos 77. México.

Pareja F. (1986). ES en el Ecuador. Centro Ecuatoriano de investigaciones Sociales (CEIS) CRESALC - UNESCO. Caracas

Rodríguez G. y Weise C. (2005) Informe Nacional de Educación Superior. IESALC- UNESCO. Quipus. Cochabamba, Bolivia.

Torres J.C. (2002) IEASLC Diagnóstico de la Educación Virtual en el Ecuador consultado en (25.01.08) http://www.utpl.edu.ec/cittes/publicaciones/archivos/ jc_torres/informe_UV_Ecuador.pdf

UNESCO (2005) Global Education Digest. Institute for Statistics. Montreal. UNESCO (2007) Global Education Digest. Institute for Statistics. Montreal.

Valdiviezo E. Patiño M. Azabache, H. (2003). Educación Superior virtual y a distancia en Perú.Consorcio de Universidades: PUCP, UL, UP, UPCH.Quito, Ecuador Consultado en (22.01.08) http://www.iesalc.unesco.org.ve

Vexler Talledo I. (2003). Informe sobre la educación superior peruana situación y perspectivas. Consultado en (17.01.08) http://www.ibe.unesco.org/International/ICE47/English/Natreps/reports/peru.pdf

Weick, K. (1976). Educational organizations as a loosely coupled system. Administrative science. Quarterly 21.

Weise, C. (2005). La Construcción de Políticas Universitarias en Bolivia. Contradicciones en una década de Desconcierto. El caso de Bolivia. Tesis para optar al grado de Magíster en Ciencias Sociales. FLACSO. Argentina.

Weise, C. (2003) La contrarreforma de Izquierda y las comunidades universitarias. Grupo Universidad y Sociedad. CLACSO. Argentina. www.clacso. edu.ar

Weise, C. (2007) Visiones de País, visiones de universidad. Cambio real o cambio aparente?. Umbrales $n^{\circ} 15$. Revista de posgrado en ciencias del desarrollo. CIDES-Universidad Mayor de San Andrés. La Paz. Bolivia

Zamalloa S. (s.f) Investigación en educación superior.

Zurita G. (2006). Informe: Educación Superior en Iberoamérica. Capítulo Ecuador. Guayaquil. 